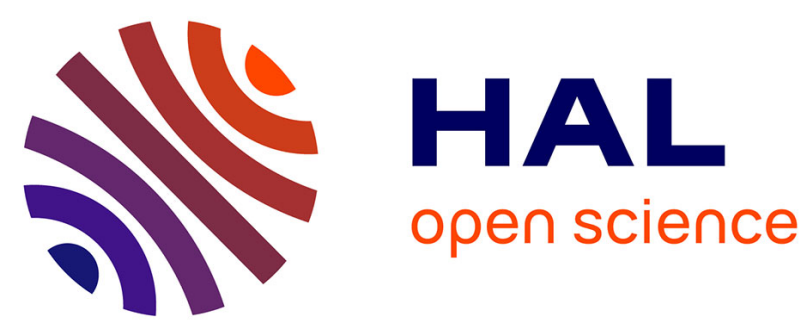

\title{
Adaptive model predictive control of the hybrid dynamics of a fuel cell system
}

Mirko Fiacchini, Teodoro Alamo, Carolina Albea-Sanchez, Eduardo Fernandez Camacho

\section{- To cite this version:}

Mirko Fiacchini, Teodoro Alamo, Carolina Albea-Sanchez, Eduardo Fernandez Camacho. Adaptive model predictive control of the hybrid dynamics of a fuel cell system. 16th IEEE International Conference on Control Applications, CCA, Oct 2007, Singapore, Singapore. pp.1420 - 1425, 10.1109/CCA.2007.4389435 . hal-00256633

\section{HAL Id: hal-00256633 \\ https://hal.science/hal-00256633}

Submitted on 15 Feb 2008

HAL is a multi-disciplinary open access archive for the deposit and dissemination of scientific research documents, whether they are published or not. The documents may come from teaching and research institutions in France or abroad, or from public or private research centers.
L'archive ouverte pluridisciplinaire HAL, est destinée au dépôt et à la diffusion de documents scientifiques de niveau recherche, publiés ou non, émanant des établissements d'enseignement et de recherche français ou étrangers, des laboratoires publics ou privés. 


\title{
Adaptive Model Predictive Control of the Hybrid Dynamics of a Fuel Cell System.
}

\author{
M. Fiacchini, T. Alamo, C. Albea and E. F. Camacho
}

\begin{abstract}
In this paper, an adaptive control scheme for the safe operation of a fuel cell system is presented. The aim of the control design is to guarantee that the oxygen ratio do not reach dangerous values. A first level of control is given by a feedforward control. An improved behavior is obtained using an adaptive predictive controller to determine the voltage to be applied to the air compressor. An admissible robust control invariant set for the PWA model of the system is computed. The control action of the predictive controller is obtained in such a way that the state is always included in the safe region characterized by the admissible robust control invariant set. This guarantees that the proposed controller always provides safe evolutions of the system.
\end{abstract}

\section{INTRODUCTION}

In the last years many research efforts have been directed to the study of hybrid systems. See, for example, [1], [2], [3], [4], [5], [6]. These systems exhibit discrete and continuous dynamics simultaneously. The presence of the two kinds of dynamics leads to the substantial inapplicability of both the classical systems theory and the automata theory.

In this paper, the application of an adaptive model predictive control to a fuel cell plant is presented. The fuel cell, located in the laboratory of the Department of Systems and Automatic of the University of Seville, generates electricity from the chemical reaction between oxygen and hydrogen. The plant is composed of different sub-systems. A complex non-linear model of the whole system has been obtained. From this model, a simple discrete-time piecewise affine (PWA) model for control purpose is identified.

In particular, the objective of the control action is to avoid that the oxygen ratio reaches dangerous values. A feedforward control of the voltage of the compressor represents a first level of control. In order to obtain a better behavior, an adaptive model predictive controller for the compressor voltage is presented. The proposed controller relies on the use of an admissible robust control invariant set. The computation of admissible robust control invariant sets for hybrid systems has been addressed in [4]. The computation of the maximal robust control invariant set for a PWA system is in many situations computationally unaffordable. Using the special characteristics of the obtained PWA system we are able to present an efficient algorithm for the computation of an admissible robust control invariant set for the fuel cell system. The presented adaptive model predictive controller incorporates a constraint that forces the

M. Fiacchini, T. Alamo, C. Albea and E.F. Camacho are with the Department of Systems and Automatic, Escuela Superior de Ingenieros, University of Seville, Spain \{mirko, alamo, calbea, eduardo\}@cartuja.us.es evolution to be confined in the obtained admissible robust control invariant set. This guarantees that in spite of the simplifying assumptions adopted to obtain an implementable adaptive predictive controller, the evolution of the system always remains in the safe region.

The paper is organized as follows. In the next section a brief description of a fuel cell is given. In section 3 the piecewise affine model of the fuel cell is presented. The computation of the admissible robust control invariant set is detailed in section 4 . The adaptive predictive controller, along with different numerical results are presented in section 5 . The paper draws to a close with a section of conclusions.

\section{Description of Fuel Cells}

A fuel cell (FC) is a device that generates electricity from hydrogen and oxygen. This is achieved by converting chemical energy of the fuel directly into electricity. A fuel cell is a class of galvanic cell based on oxidation-reduction reaction composed by three main parts:

1) Anode: where the electrons and ions are produced. The anode reaction is

$$
H_{2} \rightarrow 2 H^{+}+2 e^{-} \text {. }
$$

2) Cathode: where the ions and electrons are joined. The cathode reaction is

$$
\frac{1}{2} \mathrm{O}_{2}+2 \mathrm{H}^{+}+2 e^{-} \rightarrow \mathrm{H}_{2} \mathrm{O}
$$

3) Electrolyte: it is the electric insulator able to conduct ions. The electrolyte of the proposed Fuel Cell is a Proton Exchange Membrane (PEM) made of a polymer (Nafion).

The overall reaction is

$$
\mathrm{H}_{2}+\frac{1}{2} \mathrm{O}_{2} \rightarrow \mathrm{H}_{2} \mathrm{O}+\text { Electricity }+ \text { Heat. }
$$

See that the secondary products are merely water and heat. The rate of the reaction is determined by the electricity consumption of the external load.

The elementary fuel cell is assembled forming a Membrane Electrode Assembly (MEA) where the PEM is sandwiched between the anode, the cathode and the flow field plates. The anode and cathode are made of carbon fiber paper and a platinum catalyst. An elementary fuel cell can provide $1.2 \mathrm{~V}$ although the typical value is $0.6 \mathrm{~V}$. In order to obtain a larger voltage, fuel cells are stacked.

The fuel cell system shown in Fig. 1 is a PEM FC which operates with pure hydrogen and air and an external humidifier. This provides up to $1200 \mathrm{~W}$ of unregulated DC 


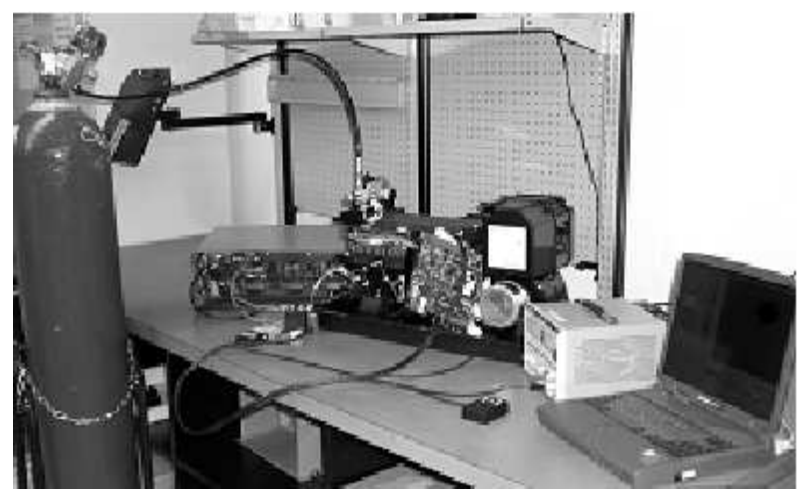

Fig. 1. The Fuel Cell

power at a nominal output voltage of $26 \mathrm{~V}$. The fuel cell is now connected to a resistive load which can be manipulated manually and will allow the simulation of several and changing loads.

The system is basically divided in five subsystems:

1) The Cell stack: it is the part of the system where the electricity is produced.

2) The electric load: simulates a real consumer of the produced electricity.

3) Air supply and humidity exchanger: the fuel cell is supplied by air with a required humidity.

4) Hydrogen storage and supply: the hydrogen pressure and flow are regulated in the operating conditions.

5) Cooling system: the heat produced by the generation of the electricity is refrigerated by a fan.

A detailed description of the fuel cell and of the related dynamic model is presented by A. Arce and A. Del Real in [7], [8]. The dynamic model of the fuel cell has been developed based on existing knowledge taken from literature (see [9]) and validated on the real plant. This model has been implemented in Matlab/Simulink environment and it has been validated with the real plant.

The oxygen ratio is a very important variable to consider due to the oxygen starvation phenomenon. The system is said to be in starvation mode when the ratio between the oxygen input flow and the reacting one is less then 2 . When it happens, the current oxygen flow can not maintain the process and this can damage the membrane and therefore destroy the fuel cell. It is clear the importance of controlling that ratio to assure that the system never enters in a starvation mode. Hence, the safety condition is

$$
\lambda_{\mathrm{O}_{2}}=\frac{W_{o_{2}, \text { in }}}{W_{o_{2}, \text { reacted }}} \geq 2,
$$

where $W_{o_{2}, \text { in }}$ is the oxygen input flow and $W_{o_{2}, \text { reacted }}$ is the oxygen reacting in the cell.

The aim of the adaptive control design is to assure that the oxygen ratio does not reach starvation values. For that purpose, the continuous-time Simulink model developed by A. Arce and A. del Real [7], [8] has been considered. A discrete-time PWA model has been obtained from this model and used to design the control, as illustrated in the following.

\section{PWA Model of THE Fuel CELl}

In the fuel cell system, the control of the air compressor is a crucial task as it is responsible for a safe operation of the system. The air compressor is controlled by means of a feedforward control. This control maintains the output $\lambda_{O_{2}}$ in a safe value for constant currents. As the variations on the compressor voltage have an important effect on the dynamics of $\lambda_{\mathrm{O}_{2}}$, this voltage will be considered as the control input for the system. The idea is to obtain, by means of an adaptive predictive controller, a control correction signal that, added to the original feedforward control, improves the performance of the system while guaranteeing that the unsafe transients are avoided.

A first analysis of the system, described in [10] (accepted at the American Control Conference, 2007), revealed a nonproper behavior of the system if the load current $I_{s t}$ was used as the input and the oxygen ratio $\lambda_{O_{2}}$ as the output. This led us to identify a first PWA model considering the ratio between the variation and the current

$$
r(k)=\frac{\Delta I_{s t}(k)}{I_{s t}(k)},
$$

as the non-manipulable input. The oxygen ratio normalized around the steady-state value was considered as the output, $y(k)=\lambda_{O_{2}}-2.2391$, and the obtained PWA model was

$$
y(k)=G_{i}(z) r(k) \quad \text { if } \quad r(k) \in R_{i},
$$

where the transfer functions depend on the active region $R_{i}$. A linear system is identified for each ratio between -0.1 and 0.1 and variation of 0.01 , that is $r_{i}=$ $-0.1,-0.09, \ldots, 0.09,0.1$. Note that the regions depend only on the input $r(k)$. Moreover, due to the PWA nature of the model, each transfer function is considered valid for a particular nominal ratio $r_{i}$ and for close values. That is, the nominal values are $r_{i}=-0.1,-0.09, \ldots, 0.09,0.1$, $i=1, \ldots, n_{i}$, where $n_{i}=21$ is the number of different regions. Hence, the distance between them is $\delta_{r}=0.01$. Furthermore, for each ratio $r$ the closest value of $r_{i}$ is considered as the nominal one. In other words, given the nominal value $r_{i}$, region $R_{i}$ is defined as $R_{i}=\left\{r:\left|r-r_{i}\right| \leq 0.5 \delta_{r}\right\}$. Note that it has been supposed that the ratio can not exceed the value of 0.105 in magnitude. It follows that the difference between the nominal ratio and the applied one is bounded, that is, denoting $\Delta_{r}(k)=r(k)-r_{i}$ with $r_{i}$ the current nominal ratio, it results that $\left|\Delta_{r}(k)\right| \leq 0.5 \delta_{r}=0.005$. Hence, the effect of this difference can be considered as a bounded additive uncertainty.

In the former model (3), the controller for the air compressor is the aforementioned feedforward law. In order to improve the control of the air compressor, a new model of the system is required. In particular, it is necessary to introduce the manipulable input which will be used as additional control signal. This new model should describe the evolution of $\lambda_{\mathrm{O}_{2}}$ as a PWA model. In the following the PWA model for control purpose is presented. It has two inputs: the 
load current (or its ratio) and the variations of compressor voltage. The ratio $r(k)$ will be considered as an external signal defining the system dynamics, that is, the active linear model of the PWA at each instant. That signal will be used in the adaptive model predictive control for selecting the linear model to employ for computing the prediction. The predictive controller provides a correction signal that is added to the feedforward law. The main objective is to compute such a correction signal in such a way that it robustly avoids the unsafe starvation region.

For this aim and knowing that the dynamics of the system depend on the the ratio $r(k)$, the PWA model has been identified applying two pseudorandom binary signal (PRBS): the first on the current ratio, around the nominal value $r_{i}$, and the second as voltage variation added to the compressor voltage.

In other words, for each nominal ratio $r_{i} \in$ $\{-0.1,-0.09, \ldots, 0.09,0.1\}$, the ratio excitation is

$$
r(k)=r_{i}+r_{P R B S}(k)
$$

where $r_{P R B S}=\left\{+\frac{\delta_{r}}{2},-\frac{\delta_{r}}{2}\right\}$ is the value of the PRBS.

The other excitation signal is the variation of the compressor voltage

$$
v_{c m p}(k)=v_{F F}\left(I_{s t}(k)\right)+v_{c}(k)
$$

where the nominal voltage $v_{F F}$ is that provided by the feedforward control and it depends only on the current, $v_{c}(k)=\{-1,+1\}$ is the added PRBS signal and the compressor voltage $v_{c m p}$ is given by their sum.

Moreover, the system is initially at the equilibrium given by $v_{c m p}(k)=v_{F F}\left(I_{s t}(k)\right)$ and the constant current $I_{s t}(k)=I_{s t}$. The output considered for identification is the value $y(k)=$ $\lambda_{O_{2}}-2.2391$ and it has been employed to identify the linear model valid around the nominal ratio $r_{i}$. The least square criterion has been applied jointly with the four pole model

$$
\begin{aligned}
y(k)= & \frac{b_{1} z^{-1}+b_{2} z^{-2}}{1+a_{1} z^{-1}+a_{2} z^{-2}+a_{3} z^{-3}+a_{4} z^{-4}} r(k)+ \\
& \frac{c_{1} z^{-1}+c_{2} z^{-2}}{1+a_{1} z^{-1}+a_{2} z^{-2}+a_{3} z^{-3}+a_{4} z^{-4}} v_{c}(k)
\end{aligned}
$$

Note that the model is given by the sum of two transfer functions whose four poles are the same. This structure provides good identification results and is very useful for computing a robust control invariant set due to its simplicity.

Then the model can be rewritten in form of regressor function, that is

$$
y(k)=\phi(k)^{T} \theta
$$

where $\phi(k)$ is the regressor composed by the last values of output and inputs and the parameter vector is $\theta=$ $\left[-a_{1},-a_{2},-a_{3},-a_{4}, b_{1}, b_{2}, c_{1}, c_{2}\right]^{T}$. The parameter vector $\theta$ is obtained solving the corresponding least square problem.

Then, for each admissible nominal ratio, a linear model is computed. As a further level of simplification we consider a PWA model composed by $n_{i}=9$ linear models, that is, the related to $r_{i} \in\{-0.1,-0.075,0.05, \ldots 0.075,0.1\}$, with $n_{i}=9$ number of regions. Hence the active linear model is the $i$-th if $r(k)=r_{i}+\Delta_{r}(k)$, with a $\left|\Delta_{r}(k)\right| \leq 0.5 \delta_{r}$, where $\delta_{r}=0.025$ and $i=1, \ldots, n_{i}$.

\section{Robust InVARIANT SET}

The first step to design the adaptive model predictive controller is to find an admissible robust control invariant set for the PWA model. This requires the computation of a region of the state-space such that, for all the contained states there is a control input such that the next state is contained in the set, for all the considered linear plants. In other words, define the state-space PWA model as

$$
x(k+1)=A_{i} x(k)+B_{i}^{R} r(k)+B_{i}^{V_{c}} v_{c}(k)+E w(k)
$$

if $r(k) \in R_{i}$ and where

$$
\begin{aligned}
A_{i} & =\left[\begin{array}{cccccc}
-a_{1} & -a_{2} & -a_{3} & -a_{4} & b_{2} & c_{2} \\
1 & 0 & 0 & 0 & 0 & 0 \\
0 & 1 & 0 & 0 & 0 & 0 \\
0 & 0 & 1 & 0 & 0 & 0 \\
0 & 0 & 0 & 0 & 0 & 0 \\
0 & 0 & 0 & 0 & 0 & 0
\end{array}\right], \\
B_{i}^{R} & =\left[\begin{array}{llllll}
b_{1} & 0 & 0 & 0 & 1 & 0
\end{array}\right]^{T}, \\
B_{i}^{V_{c}} & =\left[\begin{array}{llllll}
c_{1} & 0 & 0 & 0 & 0 & 1
\end{array}\right]^{T}, \\
E & =\left[\begin{array}{llllll}
1 & 0 & 0 & 0 & 0 & 0
\end{array}\right]^{T} .
\end{aligned}
$$

The state, normalized around the steady-state value of 2.2931, is $x(k)=\left[\lambda_{O_{2}}(k)-2.2931, \lambda_{O_{2}}(k-1)-\right.$ 2.2931, $\lambda_{O_{2}}(k-2)-2.2931, \lambda_{O_{2}}(k-3)-2.2931, r(k-$ $\left.1), v_{c}(k-1)\right]^{T}$. The active model of the PWA model is determined by the current value of the ratio $r(k)$ : the $i$-th model is valid at time $k$ if $r(k) \in R_{i}$. As the value of $r(k)$ is accessible, at each instant it is possible to know which of the 9 linear models determines the dynamics of the system.

The additive uncertainty $w(k)$ represents the error due to the difference between the PWA model and the non-linear model and it can be experimentally checked that this uncertainty never reaches amplitudes greater than 0.015 . Thus the constraint $w(k) \in \mathscr{W}=\left\{w(k) \in \mathbb{R}:|w(k)| \leq \delta_{w}=0.02\right\}$ provides an appropriate bound. The control input is bounded too: $v_{c}(k) \in \mathscr{V}=\left\{v(k) \in \mathbb{R}:\left|v_{c}(k)\right| \leq 10\right\}$

The safety condition, namely $\lambda_{O_{2}} \geq 2$, bounds the admissible region of the state space. The admissible region for the first 4 states, which are the past values of $\lambda_{\mathrm{O}_{2}}-2.2391$, is given by:

$$
2-2.2391 \leq x_{j}(k) \leq 3-2.2391 \quad j=1, \ldots, 4
$$

where the upper bound is a trivial bound never reached by $\lambda_{\mathrm{O}_{2}}$ and it is added just to simplify the algorithm.

The fifth state is the past value of the ratio. As we restrict to ratios included between $-0.1-0.5 \delta_{r}$ and $0.1+0.5 \delta_{r}$, it results that $x_{5}$ must satisfy:

$$
-0.1125 \leq x_{5}(k) \leq 0.1125
$$


The sixth state, which is the past compressor voltage, has to fulfil:

$$
-10 \leq x_{6}(k) \leq 10
$$

These linear inequalities define a safe polyhedron of the state space and can be expressed as $\mathscr{X}=\{x: M x \leq N\} \subset \mathbb{R}^{6}$.

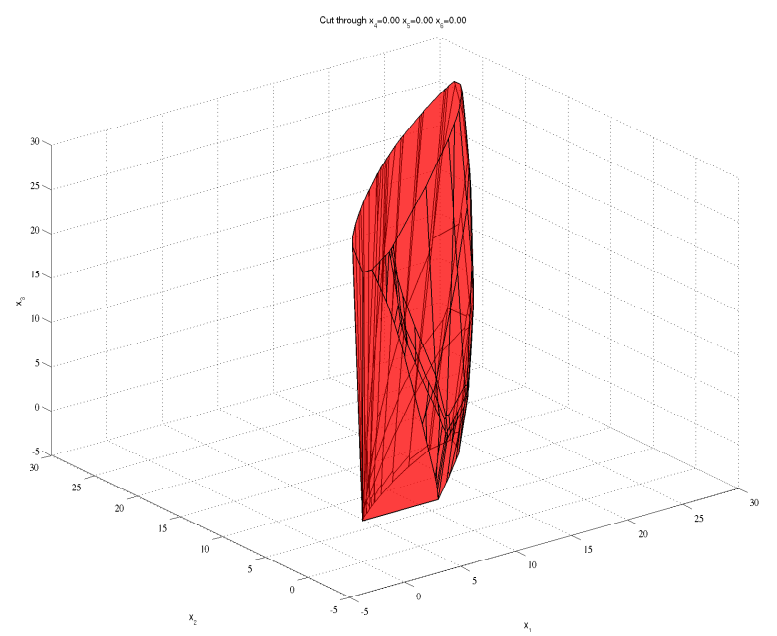

Fig. 2. Projection of the invariant set on $x_{4}=x_{5}=x_{6}=0$.

Define the set $C_{k}$ as the set of states $x$ for which there is an adaptive robust control policy that guarantees that the sequence $x(0), x(1), \ldots, x(k)$ belongs to the admissible set $\mathscr{X}$ regardless of the uncertainty and the ratio input. It is clear that $C_{k+1} \subseteq C_{k}, \forall k$. Moreover standard arguments from invariant set theory allows one to affirm that the set

$$
\lim _{k \rightarrow \infty} C_{k}=C_{\infty}
$$

constitutes an admissible robust control invariant set.

Define as a sort of extended state the vector composed by the state $x$ and the input $v_{c}$, that is: $x^{e}(k)=\left[x(k)^{T} v_{c}(k)^{T}\right]^{T}$. Given the polytopic set $\left.C_{k}=\left\{x: M_{k} x \leq N_{k}\right\}\right\}$, the set $C_{k+1}$ is given by

$$
C_{k+1}=\bigcap_{i=1}^{n_{i}} \operatorname{Proj}_{x}\left(P_{k}^{i}\right)
$$

where

$$
\begin{aligned}
P_{k}^{i}= & \left\{x^{e} \in C_{k} \times \mathscr{V}: \forall r \in R_{i}, \forall w \in \mathscr{W},\right. \\
& \left.M_{k}\left(A_{i} x+B_{i}^{V_{c}} v_{c}+B_{i}^{R} r+E w\right) \leq N_{k}\right\}
\end{aligned}
$$

and $\operatorname{Proj}_{x}\left(P_{j}^{i}\right)$ indicates the projection of the set $P_{j}^{i} \subset \mathbb{R}^{7}$ on the subspace $\mathbb{R}^{6}$ related to state $x$ and $R_{i}=\left[r_{i}-\delta_{r}, r_{i}+\delta_{r}\right]$.

Note that $P_{k}^{i}$ is the set of $x^{e}=\left[x^{T}, v_{c}^{T}\right]^{T}$ such that the state $x$ is mapped inside $C_{k}$ when the model is the $i$-th and the input applied is $v_{c}$, regardless of the admissible values of $r$ and $w$. Then, projecting $P_{k}^{i}$ on the subspace of $x$, the result is the set of states for which there exists at least an admissible value of $v_{c}$ such that the successor state is mapped inside $C_{k}$ for all $r \in R_{i}$ and $w \in \mathscr{W}$. Hence, the intersection of the projections provide the set of states that can be maintained in the safe region $k+1$ steps. Note that the previous statement relies on the fact that the value of $r(k)$ is assumed to be measurable.

The following theorem provides a way for computing $P_{k}^{i}$ given $C_{k}$. This, and equation 13 allows one to compute $C_{k+1}$.

Theorem 1: Consider the set $P_{k}^{i}$ defined in 14. Define:

$$
\begin{aligned}
S_{k}^{i}= & \left\{x^{e} \in C_{k} \times \mathscr{V}: M_{k} A_{i} x+M_{k} B_{i}^{V_{c}} v_{c} \leq\right. \\
& \left.N_{k}-M_{k} B_{i}^{R} r_{i}-0.5\left|M_{k} B_{i}^{R} \delta_{r}\right|-\left|M_{k} E \delta_{w}\right|\right\}
\end{aligned}
$$

where $\left|M_{k} B_{i}^{R} \delta_{r}\right|$ indicates the vector whose entries are the absolute values of the elements of $M_{k} B_{i}^{R} \delta_{r}$. The same for $\left|M_{k} E \delta_{w}\right|$. Then,

$$
P_{k}^{i}=S_{k}^{i}
$$

Proof: Indicate with $n_{k}$ the number of rows of $M_{k}$ and $N_{k}$. Define with $M_{k, j}$ and $N_{k, j}$ the $j$-th rows of the matrix $M_{k}$ and vector $N_{k}$, respectively. Note that:

$$
\begin{aligned}
P_{k}^{i}=\quad & \left\{x^{e} \in C_{k} \times \mathscr{V}: \forall r \in R_{i}, \forall w \in \mathscr{W}\right. \\
& \left.M_{k}\left(A_{i} x+B_{i}^{V_{c}} v_{c}+B_{i}^{R} r+E w\right) \leq N_{k}\right\} \\
=\quad & \left\{x^{e} \in C_{k} \times \mathscr{V}: \forall j=1, \ldots, n_{k},\right. \\
& \left.\max _{r \in R_{i}, w \in \mathscr{W}}\left\{M_{k, j}\left(A_{i} x+B_{i}^{V_{c}} v_{c}+B_{i}^{R} r+E w\right)\right\} \leq N_{k, j}\right\} \\
=\quad & \left\{x^{e} \in C_{k} \times \mathscr{V}: M_{k, j}\left(A_{i} x+B_{i}^{V_{c}} v_{c}\right)+\right. \\
& \left.\max _{r \in R_{i}, w \in \mathscr{W}}\left\{M_{k, j}\left(B_{i}^{R} r+E w\right)\right\} \leq N_{k, j}, \quad \forall j=1, \ldots, n_{k}\right\} \\
= & \left\{x^{e} \in C_{k} \times \mathscr{V}: M_{k, j}\left(A_{i} x+B_{i}^{V_{c}} v_{c}\right)+\max _{r \in R_{i}}\left\{M_{k, j} B_{i}^{R} r\right\}+\right. \\
& \left.\max _{w \in \mathscr{W}}\left\{M_{k, j} E w\right\} \leq N_{k, j}, \quad \forall j=1, \ldots, n_{k}\right\} .
\end{aligned}
$$

Considering that, for all $j=1, \ldots, n_{k}$,

$$
\begin{aligned}
& \max _{r \in R_{i}}\left\{M_{k, j} B_{i}^{R} r\right\}=M_{k, j} B_{i}^{R} r_{i}+0.5\left|M_{k, j} B_{i}^{R} \delta_{r}\right|, \\
& \max _{w \in \mathscr{W}}\left\{M_{k, j} E w\right\}=\left|M_{k, j} E \delta_{w}\right|,
\end{aligned}
$$

we have that

$$
\begin{aligned}
& P_{k}^{i}=\left\{x^{e} \in C_{k} \times \mathscr{V}: M_{k}\left(A_{i} x+B_{i}^{V_{c}} v_{c}\right)+M_{k} B_{i}^{R} r_{i}+\right. \\
&\left.0.5\left|M_{k} B_{i}^{R} \delta_{r}\right|+\left|M_{k} E \delta_{w}\right| \leq N_{k}\right\}=S_{k}^{i} .
\end{aligned}
$$

The following algorithm provides a way to compute an admissible robust control invariant set:

Algorithm 1:

1) Set the initial region $C_{0}=\mathscr{X}=\left\{x: M_{0} x \leq N_{0}\right\}$ and $j=0$.

2) For each region $R_{i}$ define

$$
\begin{aligned}
S_{j}^{i}=\quad & \left\{x^{e} \in C_{j} \times \mathscr{U}: M_{j} A_{i} x+M_{j} B_{i}^{V_{c}} v_{c} \leq\right. \\
& \left.N_{j}-M_{j} B_{i}^{R} r_{i}-0.5\left|M_{j} B_{i}^{R} \delta_{r}\right|-\left|M_{j} E \delta_{w}\right|\right\} .
\end{aligned}
$$

3) Compute

$$
C_{j+1}=\bigcap_{i=1}^{n_{i}} \operatorname{Proj}_{x}\left(S_{j}^{i}\right) .
$$


4) If $C_{j+1}=C_{j}$, or $C_{j+1}$ is empty then stop. Else, set $j=j+1$ and return to step 2 .

The algorithm has been applied to the obtained model and it converged after 19 iterations to a non-empty polyhedron $\hat{C}=\{x \in \mathscr{X}: \hat{M} x \leq \hat{N}\}$. The projection of $\hat{C}$ on the first three dimension is represented in Fig. 2, where $x_{4}=x_{5}=x_{6}=0$.

In the case that the obtained admissible robust invariant set would be empty or too small, a possible solution is to increase the accuracy of the PWA model, for instance using a higher order linear model or employing more regions. This would lead to a smaller uncertainty and to an enlarged robust invariant set, at the expense of a greater computational complexity.

\section{Adaptive Model Predictive Control}

The admissible robust control invariant set $\hat{C}$ is used to guarantee that the proposed controller provides a safe operation of the fuel cell. Every control strategy that forces the state $x(k)$ to remain in the admissible robust control invariant set assures that the system remains in the safe region. We employ an adaptive model predictive control strategy which minimizes a quadratic cost and guarantees that the state remains in the safe region. The model used for the prediction depends on the current ratio. Note that the use of the admissible robust control invariant set yields to a formulation of the model predictive control which guarantees robust safety for the fuel cell and is less conservative then the classic robust schemes, min-max predictive control for example.

The PWA model (9) has been used for designing the model predictive control. The inputs of the controller are the measures of $\lambda_{O_{2}}(k)$ and of the current $I_{s t}(k)$. At each instant the ratio $r(k)$ is computed from the current measure

$$
r(k)=\frac{I_{s t}(k)-I_{s t}(k-1)}{I_{s t}(k)},
$$

and the state $x(k)$ of the PWA model (8) is updated.

The active linear model is given by the value of $r(k)$ as previously described. Moreover, we suppose that the ratio is maintained constant $N$ steps, where $N$ is the control (and prediction) horizon. Note, however, that if in the prediction the current reaches the extremal values, $9 A$ and $41 A$, then the ratio is set to zero for the rest of the prediction horizon. Hence, the ratio in the prediction horizon is

$$
r(k+j)= \begin{cases}r(k) & \text { if } I_{s t}(k+j) \in[9,41] \\ 0 & \text { otherwise. }\end{cases}
$$

and $I_{s t}(k+j)$ is computed from $I_{s t}(k)$ and using equation (17).

Through a set of linear constraints, we imposed that the first predicted state belongs to the admissible robust control invariant set $\hat{C}$ despite of the uncertainties. This guarantees that the evolution of the system always remains in $\hat{C}$. The last set of constraints imposes that the predicted nominal state does not violate the safety condition $\left(\lambda_{O_{2}}>2\right)$.

The proposed model predictive control is the following:
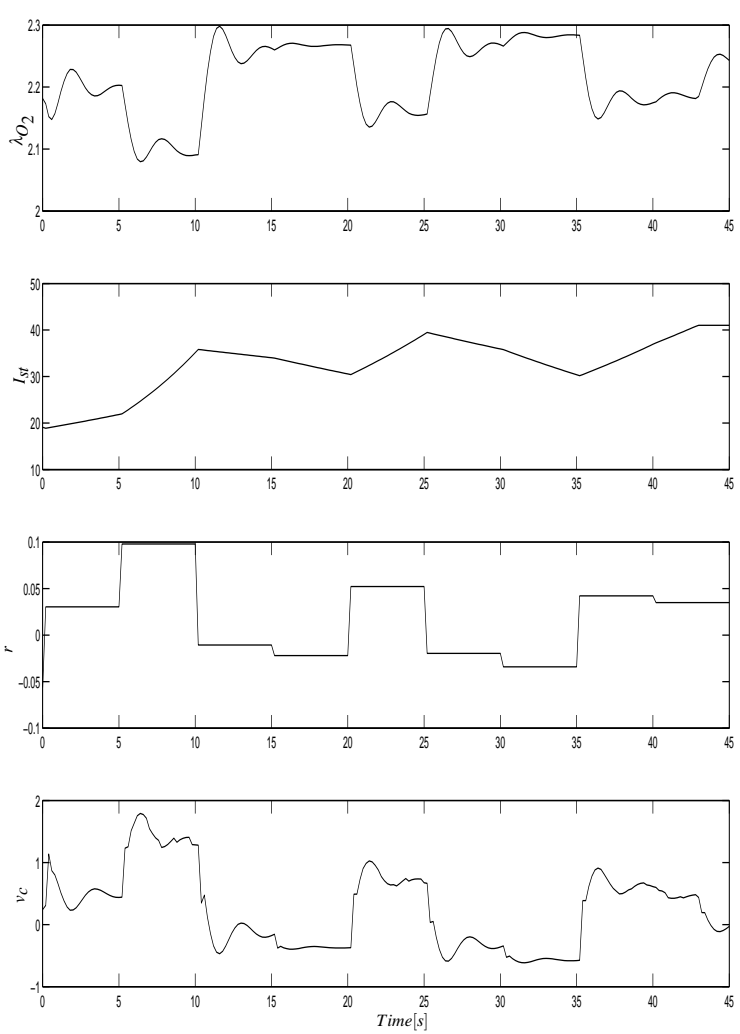

Fig. 3. Evolution with MPC and a random varying $r(k)$.

$$
\min _{v_{c}(k), \ldots, v_{c}(k+N-1)}\left\{\begin{array}{l}
\sum_{j=0}^{N-1}\left(x(k+j \mid k)^{T} Q x(k+j \mid k)+\right. \\
\left.v_{c}(k+j)^{T} R v_{c}(k+j)\right)+ \\
\\
\left.x(k+N \mid k)^{T} P x(k+N \mid k)\right\}
\end{array}\right.
$$

$$
\begin{aligned}
& \text { s.t. } \quad x(k \mid k)=x(k) \\
& \begin{aligned}
x(k+j+1 \mid k) \quad & =A_{i} x(k+j \mid k)+B_{i}^{R} r(k+j)+ \\
& B_{i}^{V_{c}} v_{c}(k+j), \\
& \text { if } r(k+j) \in R_{i}, j=0, \ldots, N-1
\end{aligned} \\
& \hat{M}\left(A_{i} x(k)+B_{i}^{R} r(k)+B_{i}^{V_{c}} v_{c}(k)\right)+\left|\hat{M} E \delta_{w}\right| \leq \hat{N}, \\
& \text { if } r(k) \in R_{i} \\
& x_{1}(k+j \mid k) \geq(2-2.2391), \quad j=1, \ldots, N .
\end{aligned}
$$

The prediction horizon is $N=8$, the cost is quadratic in the predicted state $x(k+j \mid k)$ and in the control input $v_{c}(k+$ $j), j=1, \ldots, N$. The considered output is $y(k)=C x(k)=$ $[1,0,0,0,0,0] x(k)=\lambda_{O_{2}}(k)-2.2391$ and the weighting matrices are $Q=10 C^{T} C$ for the error and $R=0.1$ for the input.

Note that, in practice, the ratio is not indefinitely maintained at a non-zero value. As a matter of fact, in normal 
operation, the ratio will be close to zero. Only in the transitory change from a load current to another the ratio will take values different from zero. Hence we consider the system corresponding to ratio zero for computing the matrix $P$. The final cost matrix $P$ is the one corresponding to the LQR obtained using the same weighting matrices.

Note that the employment of different linear models depending on the current ratio provides the adaptive nature to the control strategy. This, jointly with the safety constraint $x(k+1) \in \hat{C}$, allows to assure safeness avoiding the use of more conservative strategies, such as the min-max predictive controller.

In Fig. 3 it is shown the result of the application of the MPC in the non-linear system. The ratio $r(k)$ is constant during each interval of $5 s$ and it takes random values. The current $I_{s t}$ reaches a wide range of admissible values. The control action is smooth and it has amplitude smaller than $2 \mathrm{~V}$. Note that the value of $\lambda_{\mathrm{O}_{2}}$ never reaches the unsafe values, not even between 5 and 10 seconds, when the ratio is maintained at a value which would have caused unsafety in absence of the control.

The ability of the propose adaptive MPC to prevent oxygen starvation is more evident in Fig. 4, where the maximal value of the ratio $r=0.1125$ is maintained during 3 seconds. The value of $\lambda_{\mathrm{O}_{2}}$ does not reach unsafe values.
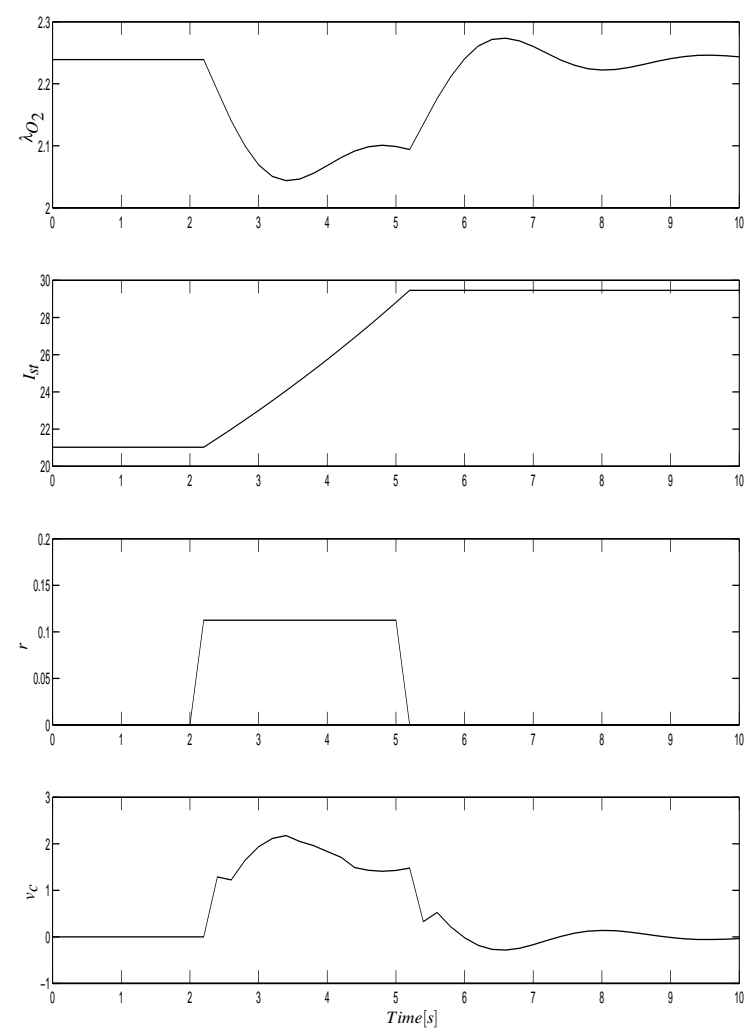

Fig. 4. Evolution with MPC and a step of $r(k)$.

\section{CONCLUSIONS}

In this paper the application of an adaptive model predictive control to a fuel cell plant has been addressed. In particular, the aim of the control action is to avoid that the oxygen ratio reaches values lower than 2 , regardless on the variation of the load current. The fuel cell plant is located in the laboratory of the Department of Systems and Automatic of the University of Seville. A simple discrete-time model in PWA form has been developed. An adaptive model predictive controller has been proposed. Such a controller relies on the computation of an admissible robust control invariant set. The controller forces the system to remain in such a safe set.

\section{ACKNOWLEDGMENTS}

The authors would like to acknowledge A. Arce and A.J. del Real for supplying the detailed fuel cell model, the European Commission and the MEC for funding this work in the framework of the NoE HYCON FP6-IST-511368 and the project DPI2005-04568.

\section{REFERENCES}

[1] W. Heemels, B. D. Schutter, and A. Bemporad, "Equivalence of hybrid dynamical models," Automatica, vol. 37, pp. 1085-1091, 2001.

[2] A. Bemporad, F. Borrelli, and M. Morari, "Optimal controllers for hybrid systems: Stability and piecewise linear explicit forms," in Proceedings of the 39th IEEE Conference on Decision and Control, vol. 2(2), Sydney, Australia, 2000, pp. 1810-1815.

[3] P. Grieder, M. Kvanisca, M. Baotic, and M. Morari, "Stabilizing low complexity feedback control of constrained piecewise affine systems," Automatica, vol. 41, pp. 1683-1694, 2005.

[4] S. Rakovic, P. Grieder, M. Kvasnica, D. Mayne, and M. Morari, "Computation of invariant sets for piecewise affine discrete time systems subject to bounded disturbances," in Proceedings of the 43rd IEEE Conference on Decision and Control., 2004, pp. 1418-1423.

[5] M. Lazar, W. Heemels, S. Weiland, and A. Bemporad, "Stabilization conditions for model predictive control of constrained PWA systems," in Proceedings of the 43rd IEEE Conference on Decision and Control, 2004, pp. 4595-4600.

[6] A. Bemporad, F. Borrelli, and M. Morari, "Piecewise linear optimal controllers for hybrid systems," in Proceedings of the American Control Conference, 2000, vol. 2, Chicago, IL, USA, 2000, pp. 11901194.

[7] A. Arce, "Modelling and control of a PEM fuel cell," Master's thesis, University of Seville, 2005

[8] C. Bordons, A. Arce, and A. Del Real, "Constrained predictive control strategies for PEM fuel cells," in Proceedings of the American Control Conference, Minneapolis, MN, USA, 2006.

[9] J. T. Prukushpan, A. G. Stefanopoulou, and H. Peng, Control of fuel cell Power Systems: Principles, Modeling and Analysis and Feedback Design. Springer, 2004.

[10] M. Fiacchini, T. Alamo, and E. F. Camacho, "Piecewise affine model of a fuel cell for safety verification." in Proceedings of the American Control Conference, New York City, NY, USA, 2007. 